\title{
Le temps du jugement
}

\section{Giovanni Careri}

\section{(2) OpenEdition}

\section{Journals}

Édition électronique

URL : http://journals.openedition.org/imagesrevues/876

DOI : 10.4000/imagesrevues.876

ISSN : 1778-3801

\section{Éditeur :}

Centre d'Histoire et Théorie des Arts, Groupe d'Anthropologie Historique de l'Occident Médiéval, Laboratoire d'Anthropologie Sociale, UMR 8210 Anthropologie et Histoire des Mondes Antiques

\section{Référence électronique}

Giovanni Careri, «Le temps du jugement », Images Re-vues [En ligne], Hors-série 1 | 2008, mis en ligne le 22 avril 2011, consulté le 30 janvier 2021. URL : http://journals.openedition.org/imagesrevues/876 ; DOI : https://doi.org/10.4000/imagesrevues.876

Ce document a été généré automatiquement le 30 janvier 2021.

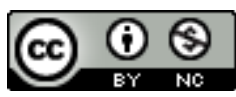

Images Re-vues est mise à disposition selon les termes de la Licence Creative Commons Attribution Pas d'Utilisation Commerciale 4.0 International. 


\section{Le temps du jugement}

\section{Giovanni Careri}

\section{1 septembre 2008}

2 La bibliographie de Michel-Ange compte environ 5000 titres dont une partie importante est consacrée à la chapelle Sixtine. Les restaurations de la voûte et du Jugement dernier ayant permis de voir ces fresques autrement, ma proposition d'interprétation en tire parti, de la même manière qu'elle s'appuie sur des études

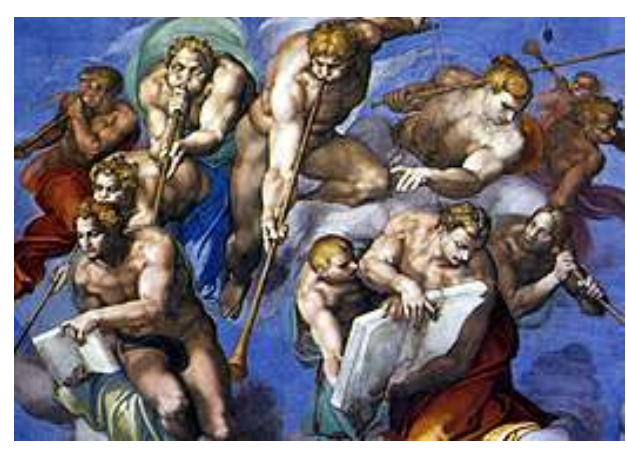
antérieures, notamment celles de Leo Steinberg ${ }^{1}$. J'y ajoute une valorisation inédite du rôle des livres de vie exhibés par les anges aux trompettes. Cela me permet de proposer pour la première fois une analyse globale de la construction de l'instance du spectateur par la fresque. Je ne me réfère pas ici au spectateur empirique, mais à l'ensemble des dispositifs internes à l'œuvre qui impliquent l'observateur en l'intégrant dans un processus de transformation ${ }^{2}$.

3 Pour aborder cette complexe construction, j'analyserai dans un premier temps la structuration des temps dans la fresque: l'aspectualisation de la temporalité sera considérée comme une façon d'impliquer l'actant observateur dans l'action figurée ${ }^{3}$. Du point de vue de la théologie chrétienne, le temps du Jugement dernier est le temps dans lequel la deuxième venue du Christ clôt le temps humain. Le déclenchement du temps dernier, le temps de l'eschaton, est caractérisé par la parfaite contemporanéité de "l'être présent du Christ », sa parousie, de la résurrection des corps et de l'opération du jugement qui sépare les élus des damnés. 
Fig.1

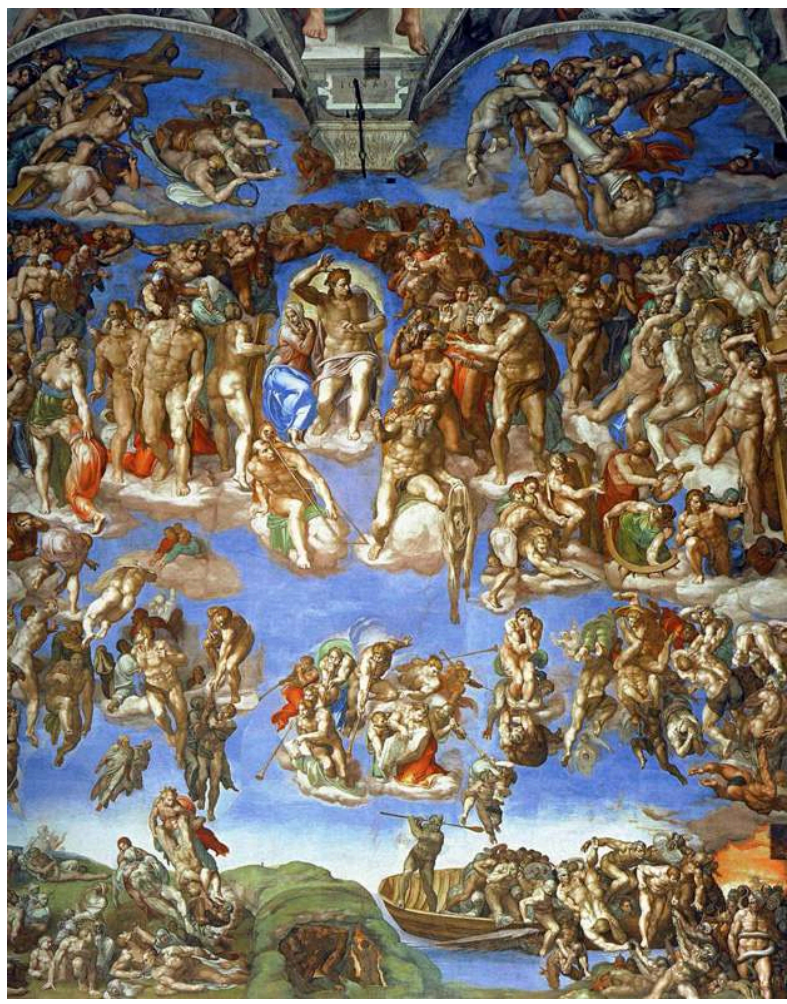

Michel-Ange, Jugement dernier, 1541, Vatican, chapelle Sixtine

4 Dans la fresque de Michel-Ange, la figuration de ce temps dernier parvient à articuler ces opérations dans un seul processus, selon la catégorie aspectuelle de l'inchoativité. Le geste du Christ déclenche le procès de la résurrection et du jugement; les très nombreuses postures d'étonnement de la part de ceux qui ressuscitent, comme aussi des élus, sont les marques passionnelles qui contribuent à construire la dimension inchoative de la temporalité de la fresque (fig.1). Cette configuration aspectuelle implique un observateur frappé, lui aussi, de stupeur, en état d'attente : l'attente du déroulement du processus à peine déclenché, mais aussi, nous le verrons, l'attente « accélérée » de son propre jugement. 
Fig. 2

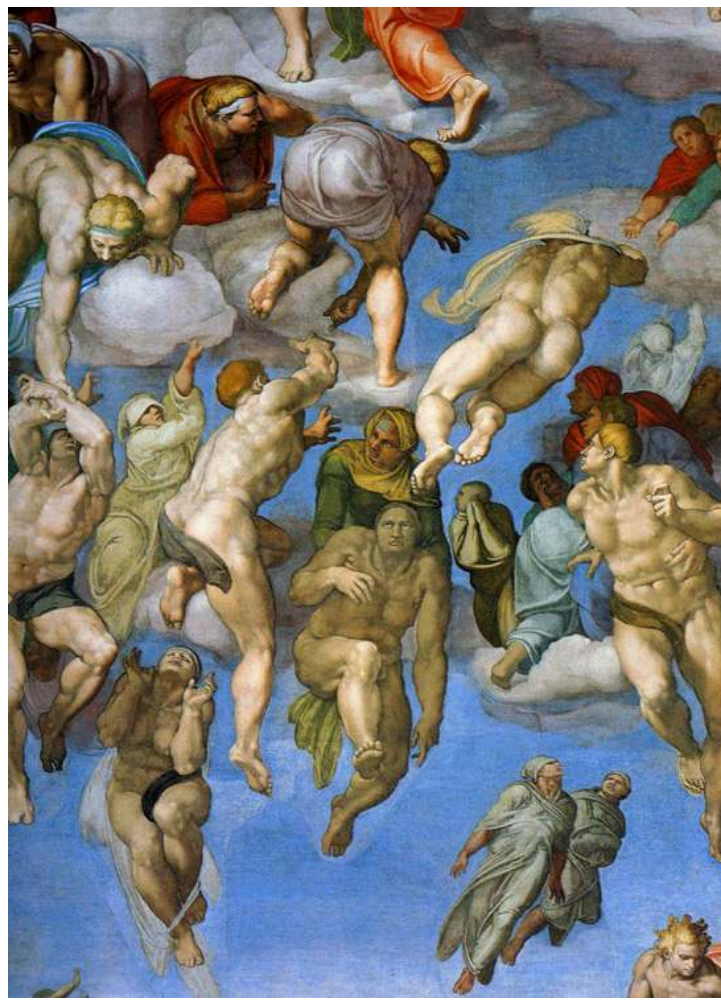

Michel-Ange, Jugement dernier, 1541, Vatican, chapelle Sixtine (détail)

5 Les marques temporelles de la configuration aspectuelle du Jugement dernier sont définies par l'articulation des postures des corps et des affects correspondants. Par leur organisation sérielle, ces « amas de corps » construisent un ensemble d'actions en cours qui dilatent le temps en autant de procès de brève durée. Je suis tenté d'utiliser la métaphore spatiale de l'épaisseur du temps car ces actions creusent des zones d'épaisseur dans la surface peinte de la fresque, tout comme elles produisent des moments d'articulation et d'expansion à l'intérieur de l'action principale. Ainsi, par exemple à la gauche des deux élus qu'un ange soulève à l'aide d'un rosaire se trouve un groupe de six figures disposées sur une petite surface selon une organisation par strates d'une densité inédite dans l'histoire de la peinture (fig.2). Cette spatialité, proche de certains bas-reliefs de Donatello, et de Michel-Ange lui-même, valorise la dimension collective de la transformation en cours: elle est figurée à travers les passages entre des postures corporelles connexes et extrêmement variées plutôt que dans une configuration hiérarchique stable disposée dans un espace organisé et intelligible ${ }^{4}$.

6 Le son des trompettes des anges marque la détermination auditive de la dimension inchoative de l'action : le début de la fin des temps a la durée, l'intensité et la vitesse d'un souffle. Bien qu'exposé au spectacle de la fin du temps, le spectateur demeure dans le temps de l'attente, dans le temps qui reste, le temps messianique situé entre le temps chronologique et l'eschaton apocalyptique de la fin.

7 Dans son commentaire de l'Epître aux Romains de saint Paul, Giorgio Agamben définit le temps messianique paulinien comme "un temps qui se contracte et qui commence à finir $»^{5}$. À titre d'hypothèse générale, j'avance l'idée que le Jugement dernier de MichelAnge est conçu comme un dispositif pictural efficace de contraction du «temps qui 
reste ». Saint Paul désigne ce temps résiduel par l'expression ho nun kairos, le temps de maintenant qui est le temps de la récapitulation et de la conversion. Mon interprétation de la fresque est tout entière orientée par la tentative d'analyser et de décrire les figures efficaces de la récapitulation et de la conversion qui visent à déterminer une forme de transformation et d'assujettissement du spectateur par l'image.

La construction temporelle du Jugement dernier peut être introduite par l'analyse du geste du Christ. Interprété de façon univoque par Giorgio Vasari ${ }^{6}$ comme expression de colère et de condamnation, il à été décrit par Ascanio Condivi comme un geste double, expression de colère certes, mais aussi de douceur: "Au dessous des Anges aux trompettes il y a le Fils de Dieu en majesté, le bras et la puissante droite levée, tel un homme qui en colère maudit les damnés et les éloigne de sa face vers le feu éternel, alors qu'avec la main gauche étendue vers la partie droite il semble recueillir les bons avec douceur $»^{7}$.

\section{Fig.3a et fig.3b}

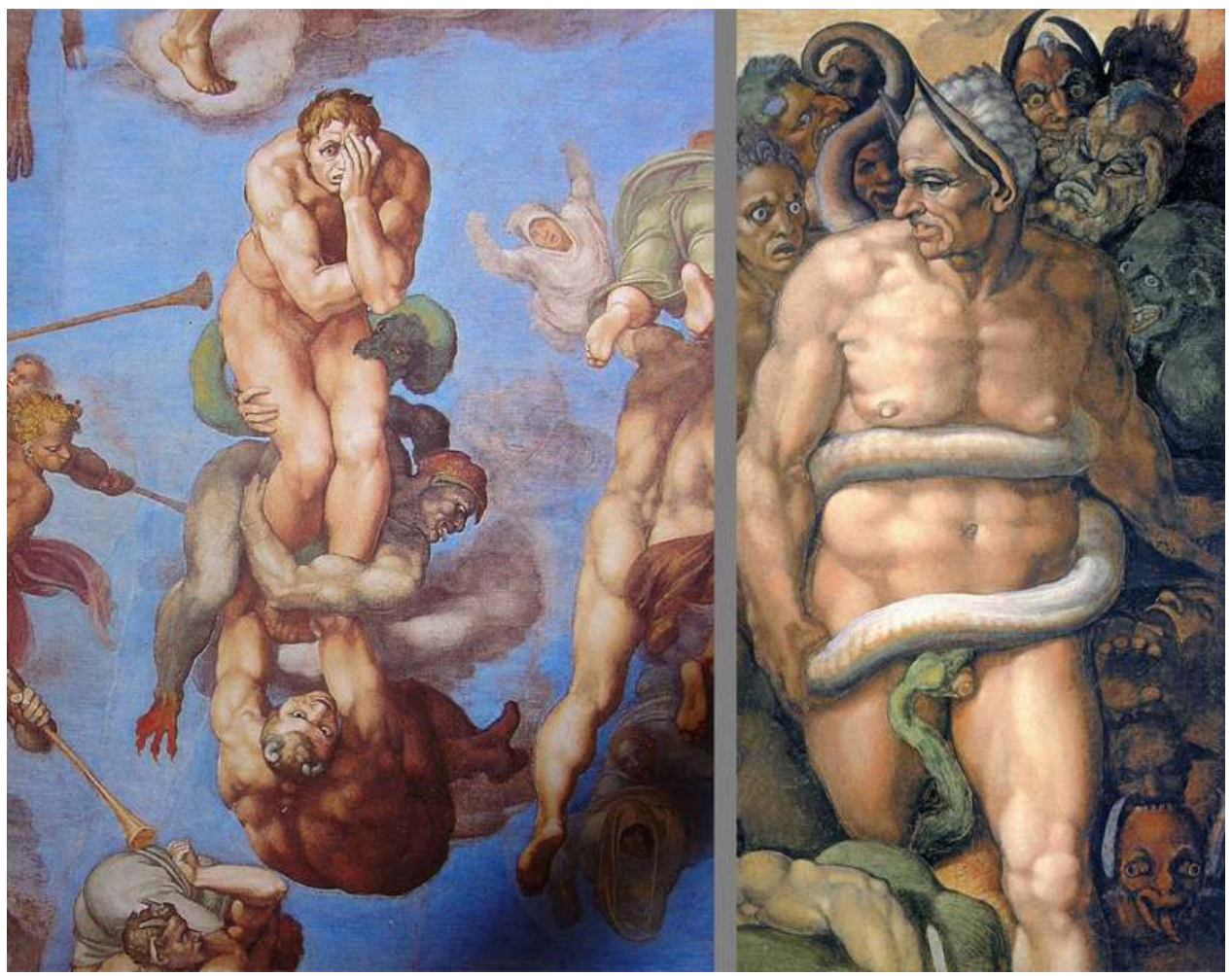

Michel-Ange, Jugement dernier, 1541, Vatican, chapelle Sixtine (détails)

La description de Condivi renvoie très précisément à un passage de la lettre de saint Paul aux Thessaloniciens où, en citant Isaïe, il décrit la damnation comme la perte de la vue du visage du Christ ${ }^{8}$. Cette privation s'accompagne de la perte définitive de la ressemblance au Christ. A l'envers l'élection introduit à la vue du visage du Seigneur. L'acte de « recueillir les bons » correspond donc à l'opération qui transforme les corps des élus en les conformant au corps du Christ. Nous allons voir comment le processus d'élévation s'articule au processus de " prise de ressemblance » et à son envers, mais en faisant recours à la description du geste du Christ par Condivi, on peut déjà commencer à regarder l'ensemble de la surface de la fresque comme un champ où s'affrontent deux 
forces. Les formes et notamment les formes enchevêtrées des corps, incarnent le déploiement de ces forces opposées. Il devient alors possible de relever un type d'organisation de l'espace et du temps fondé sur une partition selon l'intensité du conflit et selon les transformations morphologiques produites par l'augmentation ou la diminution de l'une des deux forces. Cette organisation bipolaire et morphogénétique associe le mouvement d'élévation ou de chute à une transformation par ressemblance ou par dissemblance avec le Christ. Ainsi, dans la partie médiane de la fresque, le conflit des forces est plus intense et on peut décrire les postures des anges sans craindre d'aller à l'encontre de la théologie comme les formes qui figurent la force émanant de la volonté du Christ. Aux deux extrémités du Jugement dernier se situent, comme nous le verrons, les formes les plus ressemblantes en haut et les plus dissemblantes, en bas. La figure de Minos est le pôle attractif négatif dans ce champ de forces. Le damné qui chute vers le démon est pris dans un procès de prise de ressemblance au diable que nous pouvons nommer, en renversant l'expression de saint Paul, "conformation négative » (figs.3a et $b$ ).

10 Cependant, si tel était le seul champ de forces actif dans la fresque, nous aurions affaire à une dynamique qui exclue totalement d'autres volontés et d'autres forces que celle du Christ. Or la singularité du Jugement de Michel-Ange est d'attribuer à l'homme une volonté qui, tout en étant exposée et soumise à la volonté du Christ, conserve une marge d'autonomie. La situation paradoxale de cette volonté, libre et soumise à la fois, doit être comprise en termes d'adéquation entre le jugement du Christ et le jugement de soi. Adéquation qui fait du Jugement dernier de Michel-Ange une sorte d'incunable de la subjectivité moderne dans la mesure où elle donne figure à l'intériorisation par le sujet du pouvoir qui le gouverne'.

11 Deux constellations de figures expriment la convergence de la force du jugement qui vient de l'extérieur avec celle qui monte de l'intérieur : la première se déploie autour des Livres des vies exhibés par les anges, la deuxième est organisée par la scène dantesque du bateau de Charon.

La description qu'Ascanio Condivi donne des Livres des vies estune nouvelle correction de la description donnée par Vasari, signe probable d'une intervention de Michel-Ange lui-même. Vasari évoque les anges de l'Apocalypse de saint Jean, il insiste sur la terribilità des anges de Michel-Ange et décrit les livres qu'ils montrent comme « le livre des vie $~^{10}$. Condivi ajoute : "...au milieu de l'air, près de la terre sont les sept Anges décrits par saint Jean dans l'Apocalypse, lesquels, les trompettes à la main, appellent les morts au jugement depuis les quatre parties du monde, et parmi ceux-ci deux autres, un livre ouvert à la main, dans lequel chacun en lisant et en reconnaissant la vie passée a presque à se juger soi même $»^{11}$.

13 Le commentaire insiste sur le caractère individuel du jugement: le livre s'adresse à chacun, à l'intérieur et à l'extérieur de la fresque. L'invitation signifiée par ces pages blanches est remarquable puisqu'elle ouvre un espace à la volonté de chaque individu : volonté de se constituer comme sujet d'élection ou de damnation par une opération de mémoire. Commentant saint Paul, Origène décrit la page du Livre de vies comme une surface où ce qui constitue le sujet dans le secret de son âme, accède à la visibilité de tous dans l'instant de la fin du temps: «Les livres dont parle le prophète Daniel, et qui doivent être ouverts au tribunal de Dieu pour devenir lisibles à toute créature raisonnable c'est la série de nos pensées et de nos actes, gravés au plus profond de notre cœur $»^{12}$. Le fait que les livres peints par Michel-Ange soient blancs marque 
l'imminence de l'achèvement de l'opération de mémoire et de l'inscription, ces livres sont montrés à l'intérieur comme à l'extérieur de la fresque et constituent donc un dispositif d'implication du spectateur. Plus exactement, ils définissent son opération de regard en termes de conversion au double sens d'une conversion du regard de l'extérieur vers l'intérieur et de conversion comme conséquence de l'examen de conscience dans l'attente du jugement par le Christ ${ }^{13}$.

Le Jugement Dernier ouvre un temps opératif, à savoir « une transformation actuelle de l'expérience du temps qui soit capable d'interrompre ici et maintenant le temps profane $»^{14}$, cette interruption est décrite par Saint Augustin très précisément en termes de mémoire : «Il faut donc admettre une certaine force divine sous l'action de laquelle seront évoquées à la mémoire de chacun toutes ses œuvres, et les bonnes et les mauvaises, et toutes seront saisies par un regard de l'esprit d'une étonnante rapidité ; cette connaissance accusera ou excusera la conscience et ainsi, en même temps, tous et chacun seront jugés " $^{15}$. Et il ajoute : «C'est évidemment cette force divine qui a reçu le nom de livre. De fait, en elle on lit en quelque sorte tout ce qu'on se rappelle sous son action $»^{16}$. La définition des livres montrés par les anges le jour du jugement comme forme d'une force qui pousse à lire en soi même "tout ce qu'on se rappelle » nous encourage à poursuivre notre analyse selon le modèle « morphogénétique » à savoir en cherchant à saisir le travail des formes comme l'effet du déploiement d'une ou plusieurs forces. Certes, ce bref extrait de La Cité de Dieu mériterait un long commentaire car il évoque la théorie augustinienne de la mémoire ainsi que le rôle de la lecture dans la constitution du sujet et dans sa conversion ${ }^{17}$. Dans cet article cependant nous allons tenter de faire dialoguer avec la fresque seulement les éléments pertinents à la compréhension de la peinture en respectant son autonomie. Selon cette perspective, je privilégie systématiquement toute source écrite qui puisse permettre de construire les schémas anthropologiques fondamentaux susceptibles d'éclairer le fonctionnement des processus de transformation tels que la peinture les représente et les produit. Ainsi, au lieu de cheminer dans une élaboration de la philosophie de la mémoire chez Augustin, je me propose de repérer dans la fresque elle-même les formes picturales spécifiques de la figuration de la remémoration du jugement de soi induites par la monstration de Livres des vies. 
Fig.4

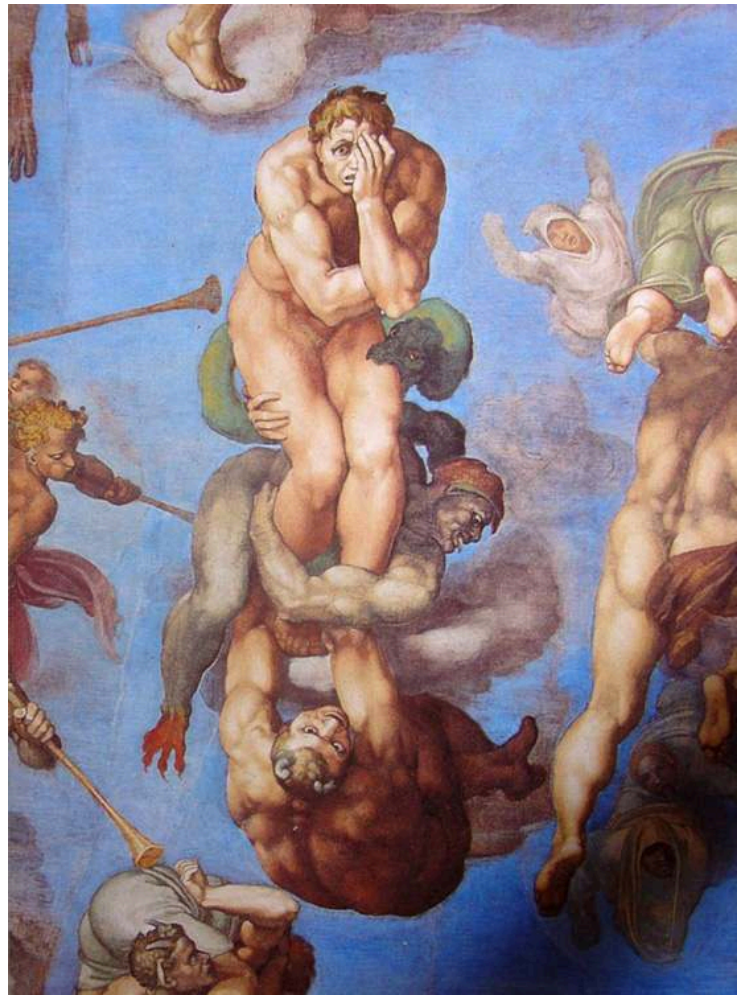

Michel-Ange, Jugement dernier, 1541, Vatican, chapelle Sixtine (détail)

15 La grande figure du "désespéré ", le personnage mordu par un serpent situé dans la zone médiane de la fresque à droite des anges aux trompettes porte dans sa posture tous les signes de la mélancolie et de l'introspection (fig.4). À ma connaissance, ce personnage n'a jamais été mis en relation avec l'invitation au jugement de soi avancée par les livres alors qu'il constitue une image saisissante de l'opération de mémoire qui accuse le pécheur et le condamne.

Placé de face par rapport au spectateur, le « désespéré » est le miroir de ses remords. C'est précisément au rimorso que l'image semble renvoyer à la fois par ses composantes mélancoliques et par les morso, la morsure mot intégré à l'italien rimorso qui dit une deuxième morsure, la condamnation de soi qui répète et confirme la condamnation divine.

17 Pour notre analyse, il est important de noter l'harmonisation et la simultanéité entre l'auto condamnation et la condamnation par le Christ: le "désespéré " "accuse sa conscience " alors qu'en même temps trois démons viennent le capturer et le tirer vers le bas. Leurs corps composent en une figure trois éléments : un serpent, un démon aux semblances humaines, et une créature hybride. Les spires du serpent se poursuivent dans les bras livides du démon hybride et dans les bras musclés du démon qui se bascule aux pieds du désespéré. La composition de cette figure serpentine déclenche le processus de prise de ressemblance du " désespéré » à Minos et par ce biais l'amorce du procès de métamorphose de l'humain vers l'animal dont les trois créatures qui entourent le « désespéré » sont les figurations. 
Fig.5a et fig.5b

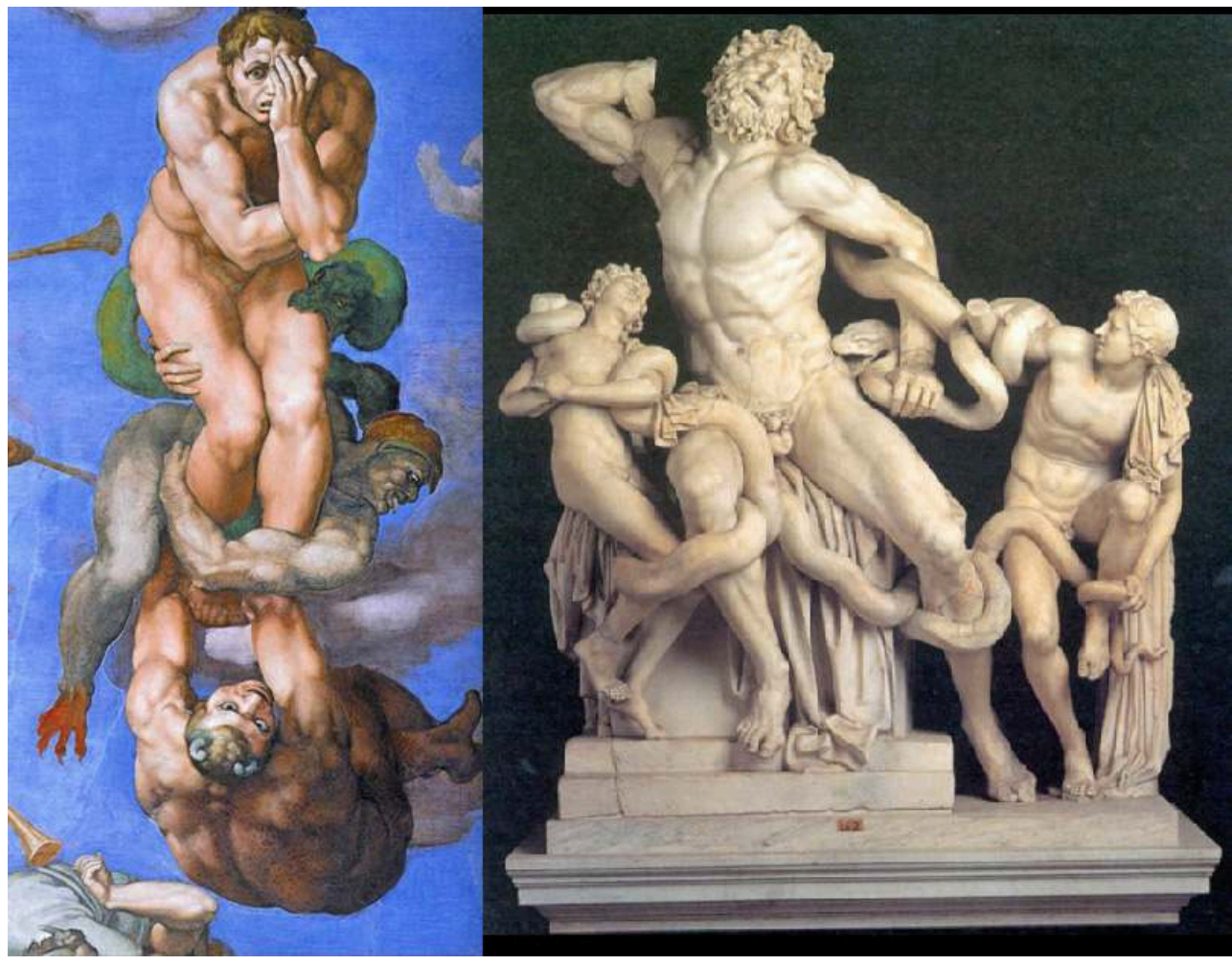

5a. Michel-Ange, Jugement dernier, 1541, Vatican, chapelle Sixtine (détail), 5b. Agésandre, Polydore et Athénodore, Le Laocoon (c. 50 av. J.-C.), Rome, Musée du Vatican

Selon la conception humaniste du châtiment que Leo Steinberg a repéré dans la fresque de Michel-Ange, l'enfer perd le caractère de la douleur physique pour se constituer comme lieu de la conscience investie par le remords ${ }^{18}$. La honte et la culpabilité, que Pietro Pomponazzi, dans son Trattato sull'immortalità dell'anima (1516), qualifie de "peines essentielles» sont les tourments auxquels les damnés sont soumis ${ }^{19}$. La punition du "désespéré » est "la mortification psychique », selon l'expression de Leo Steinberg, dans ma perspective elle devrait plutôt être décrite comme «auto/ mortification psychique». Les liens entre cette intériorisation de la faute et la constitution du sujet moderne telle que Michel Foucault l'a analysée sont évidents. Cette mutation du statut du sujet trouve une forme d'explicitation dans la reprise de la figure du Laocoon, signalée par l'encerclement, les formes serpentines et la morsure de la cuisse droite du désespéré (figs.5a et b). La formule pathétique du Laocoon hellénistique porte dans la figure du damné la marque visuelle du destin tragique. Chez le désespéré cependant la condition tragique n'est pas la conséquence d'une décision insondable des dieux, comme pour le prêtre troyen, mais l'effet de l'intériorisation chrétienne de la faute. Placé de face par rapport au spectateur, l'homme mordu est le miroir de son remords. Et c'est effectivement au rimorso que l'image semble renvoyer à la fois par ses composantes mélancoliques et par le morso; la morsure,mot intégré à l'italien rimorso qui dit une seconde morsure, c'est la condamnation de soi qui répète et confirme la condamnation divine. 
Fig. 6

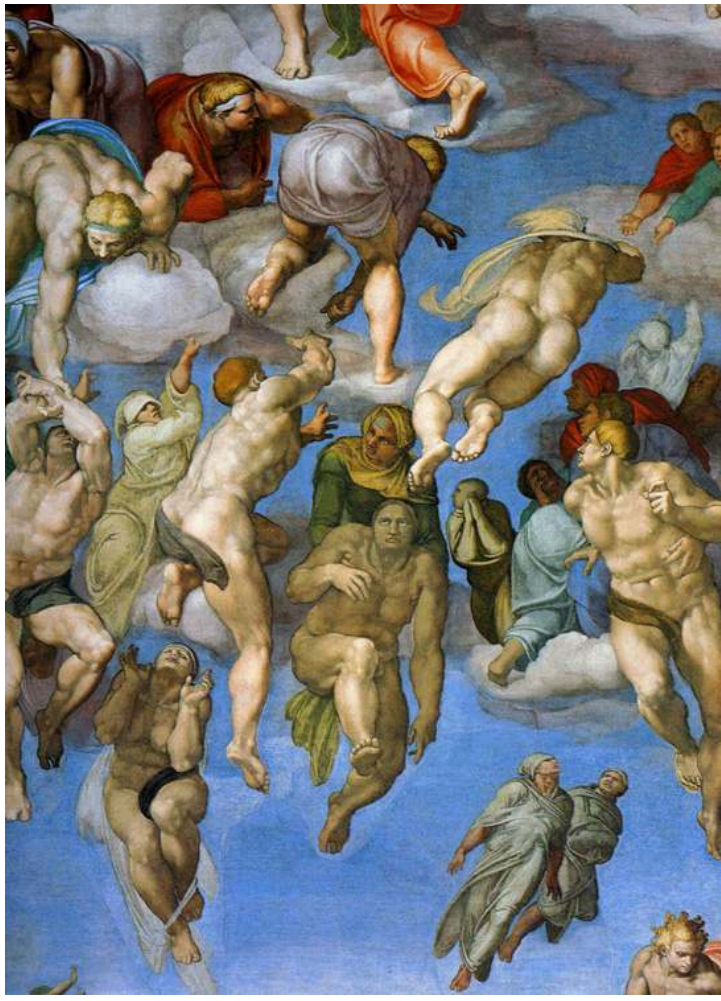

Michel-Ange, Jugement dernier, 1541, Vatican, chapelle Sixtine (détail)

Par sa frontalité, la figure de l'élu transporté au ciel par un ange situé de l'autre côté de la fresque peut être vue comme le pendant positif du «Laocoon chrétien " (fig.6). En état d'absence à soi-même, il est "ravi» au ciel. L'auto/jugement qui «excuse sa conscience » et le sauve est en train de s'accomplir et déjà une force extérieure l'élève et l'assujettit. Son corps demeure pourtant désarticulé, incapable d'organiser les rapports entre les membres, comme à signifier un état infantile: une nouvelle naissance. L'élu extatique incarne la renaissance du corps ressuscité, le tout premier moment du processus de conformation au corps glorieux du Christ. À l'opposé du remord qui mine l'unité du sujet et le voue à la scission, cette figure constitue pour le spectateur l'amorce phantasmatique de son unification dans le processus de ressemblance à l'aimé. L'opération de jugement de soi est en effet concomitante avec celle de la résurrection des corps ainsi que le précise saint Paul : « Notre Seigneur Jésus Christ reformera le corps de notre humilité en le conformant à son corps glorieux, par cette vertu efficace par laquelle il peut s'assujettir toute chose $»^{20}$. Si nous associons la force qui pousse au jugement à celle qui transfigure les corps ressuscités nous pouvons en déduire que dans la fresque on pourra reconnaitre les élus par leur participation à un processus qui les transfigure à l'image du Christ. Les damnés, de leur côté seront pris dans un processus de "prise de dissemblance ». Dans la fresque, la conformation des élus correspond à l'idéalisation de leurs corps, mais aussi et surtout, à leur participation enthousiaste et différenciée au processus de transfiguration lui-même. La déformation des damnés va à l'encontre de ce processus d'idéalisation, elle suit un procès inverse d'animalisation par lequel ils deviennent des corps monstrueux. 


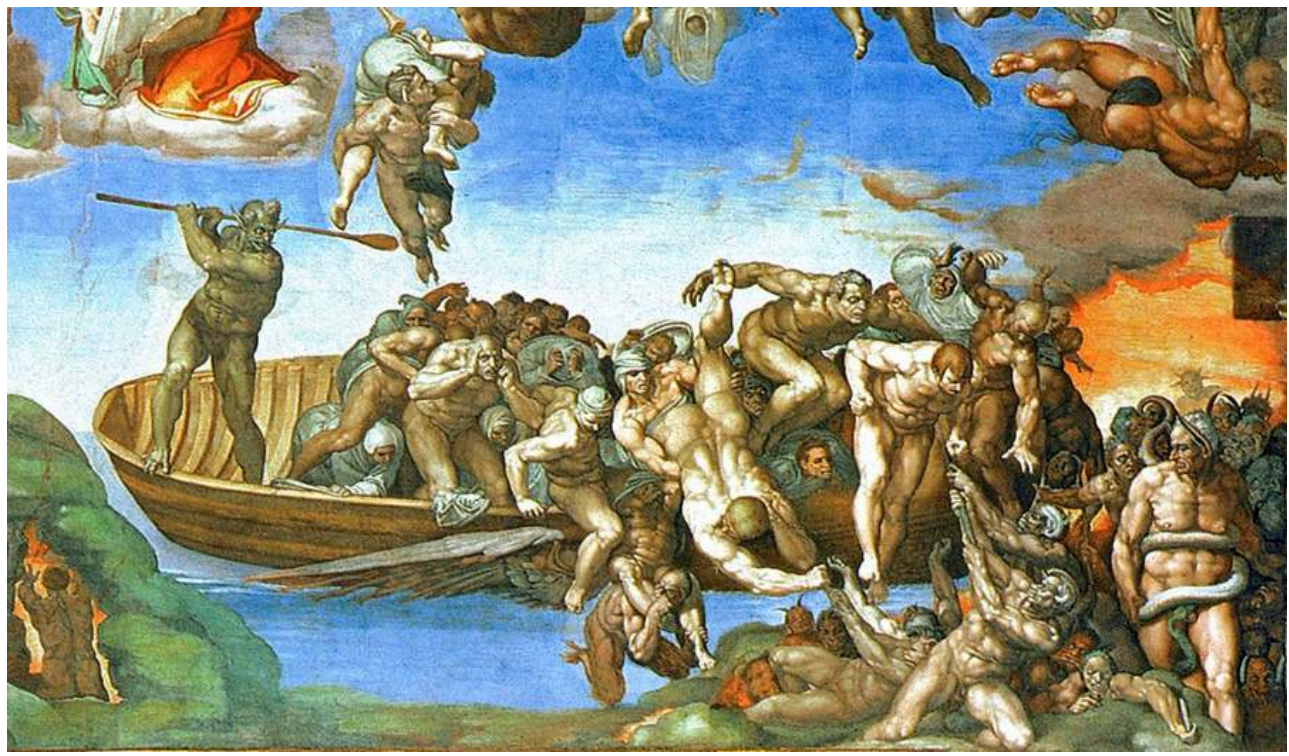

Michel-Ange, Jugement dernier, 1541, Vatican, chapelle Sixtine (détail)

Le lien entre l'action des damnés, le jugement du Christ et le jugement de soi se manifeste aussi dans le choix de la scène dantesque que Condivi décrit, encore une fois, de façon plus précise que Vasari (fig.7) Alors que ce dernier cite les vers qui décrivent l'action de Caron, Condivi se réfère à un vers qui concerne directement le rapport entre les deux jugements.

"Figliol moi", disse il maestro cortese,

"Quelli che muoion nell'ira di Dio,

Tutti convegnon qui d'ogni paese;

E pronti sono a traversar lo rio,

che la divina giustizia li sprona,

si che la tema si volge in desio".

"Mon fils", dit le maître courtois

"ceux qui meurent dans la colère de Dieu

arrivent ici de tous pays;

et ils sont prêts à traverser le fleuve,

car la divine justice les presse,

et leur peur se change en désir"21.

Ces vers sont ainsi repris par Condivi dans sa description de la fresque: «Sous les damnés on voit Caron avec son petit bateau, telle que le décrit Dante dans son Enfer dans le marais d'Hachérons. Il lève la rame pour battre toute âme qui se montre lente. Et une fois le bateau arrivé sur la berge on voit toutes les âmes se jeter hors du bateau, poussées par la justice divine de sorte que la peur, comme dit le poète, se change en désir $»^{22}$.

La crainte transformée en désir correspond à la reconnaissance inéluctable de la justice divine et en conséquence à la mise en acte immédiate du jugement de soi. Les damnés qui font face à Caron figurent par leurs gestes la tema, la crainte. À l'extrémité opposée du bateau se manifeste le desio, le désir de se jeter sur le rivage. Les damnés plongent sur le rivage de l'Hacherons en se conformant à l'impulsion de la giustizia divina qu'ils ont assumée comme la leur. Entre ces deux groupes s'opère la transformation; le renversement du volgere; l'action de se détourner de la tema vers le desio. Ce passage est 
figuré par les gestes de cinq damnés dont l'un, mélancolique, doit être associé dans l'introspection désespérée de soi à la figure du damné mordu par un serpent qui se trouve au-dessus de lui. Une autre figure, complètement encapuchonnée, représente la douleur absolue selon la tradition plinienne, un troisième personnage hésite avant de descendre du bateau, un autre encore essaie en vain de retenir un plongeur qui a déjà attrapé la corde que lui tend un démon.

Si maintenant nous essayons de rendre visible l'opérativité figurative globale du principe de la conformation par une analyse formelle de la fresque, nous pouvons considérer la posture du Christ comme la matrice formelle des attitudes de tous les corps représentés. En effet, comme l'écrit Pierluigi de Vecchi, en absence de toute partition architectonique des espaces, seule la « syntaxe des corps » tient ensemble les parties de la fresque ${ }^{23}$. L'agencement des postures corporelles constitue par ailleurs une alternative à la syntaxe gestuelle nécessaire à l'historia selon Alberti. Alors que ce dernier souhaite que les corps prennent place dans un espace organisé par la perspective en fonction d'une articulation narrative régie par la concinnitas et la convenientia, la fresque de Michel-Ange semble neutraliser la profondeur, sauf dans des sections localisées en bas. Comme ses critiques l'ont relevé on n'y reconnaît ni harmonie ni décorum. On peut d'ailleurs se demander dans quelle mesure cette image de la fin du temps humain est une peinture d'histoire au sens albertien. Centrée autour de la figure du Christ, la fresque semble plutôt venir en avant dans l'espace du spectateur que l'inviter à entrer dans son espace. En cela elle retrouve dans une certaine mesure la dimension "épiphanique " de l'icône médiévale. Elle relève plutôt d'une figuration du présent ou d'une présence que de la construction rhétorique d'un récit avec son début, son milieu et sa fin. Le problème formel que l'œuvre semble affronter est celui de l'articulation et de la propagation d'un seul mouvement dans l'épaisseur temporelle d'un seul instant. Tout en étant conforme à la figuration traditionnelle du jugement dernier, la centralité du corps du Christ et sa frontalité peuvent se comparer avec les tentatives mises en œuvre par Michel-Ange pour réactualiser l'imago pietatis comme dans le cas de la mise au tombeau de Londres étudiée par Alexander Nage ${ }^{24}$.

Formellement, le geste du Christ du Jugement dernier est à la fois un contrapposto et une figura serpentinata, une serpentine. Le Contrapposto, opposition contrastive de deux forces en une figure, est une notion que la théorie de l'art tire de la rhétorique où elle est associée à l'antithèse. Nous avons vu que les deux bras du Christ signifient et déterminent le déploiement de deux forces opposées. L'articulation des formes qui figurent ces forces est le principe d'intelligibilité de l'image qui remplace le dispositif de la perspective. La notion de figura serpentinata relève de la langue spécialisée des artistes et de Michel-Ange en particulier. Elle décritle contrapposto en termes de mouvement: "On dit donc que Michel-Ange donna à Marco da Siena, peintre et disciple, ce précepte : qu'il fasse toujours la figure pyramidale, serpentine et multipliée par un deux ou trois. Et il me semble que dans cet enseignement consiste tout le secret de la peinture. Car la grâce et la beauté la plus grande qu'une figure puisse avoir est qu'elle se montre en mouvement, ce que les peintres nomment la furia de la figure. Et pour représenter ce mouvement il n'y pas de forme plus adaptée que la flamme du feu [...] et le peintre doit accompagner cette forme pyramidale avec la forme serpentine qui représente le mouvement recourbé d'un serpent vivant quand il marche, ce qui correspond à la forme de la flamme du feu qui se meut comme une vague $»^{25}$. 


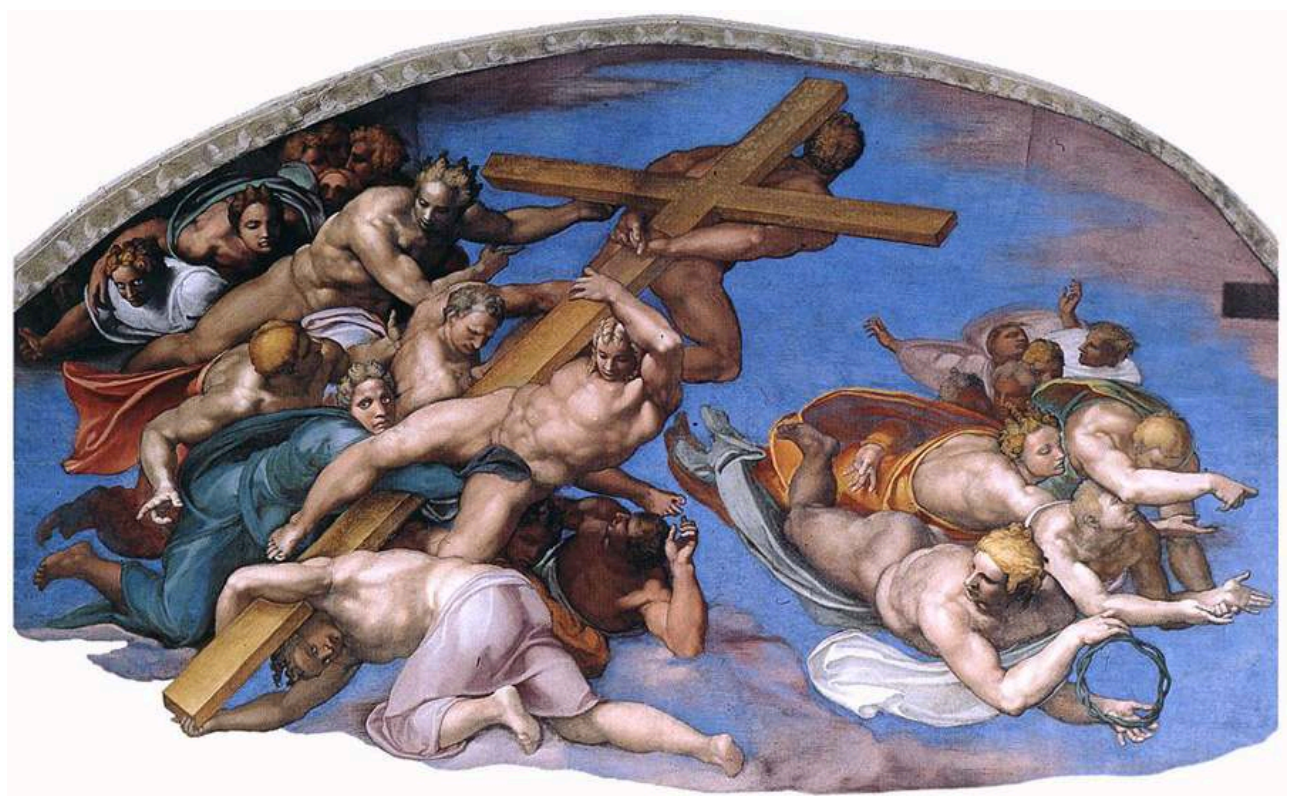

Michel-Ange, Jugement dernier, 1541, Vatican, chapelle Sixtine (détail) ondulatoire d'un mouvement qui reproduit sa propre forme dans un processus de variation formelle et énergétique. Comme la flamme et la vague, la figura serpentinata du geste du Christ est la matrice des postures des autres figures et le principe formel de leur syntaxe. C'est l'élément à partir duquel se diffuse un mouvement qui donne forme. La conformation au Christ doit donc être vue sous l'aspect de l'assomption du mouvement de torsion de sa posture. De ce point de vue - morphogénétique - les anges qui portent les instruments de la Passion ressemblent au corps glorieux du Christ dans la mesure où ils assument la torsion serpertine de sa figure et la portent à son point le plus extrême (fig.8). Cette torsion correspond d'ailleurs à l'agilitas qui, d'après les textes canoniques de saint Thomas, caractérise les corps des anges et des élus après le Jugement dernier. Du point de vue morphologique, la fresque peut donc être considérée comme un champ de forces global qui inclue des phénomènes conflictuels globaux et locaux. La zone intermédiaire, là où les anges aident les élus à monter au ciel et repoussent les damnés est celle où la force d'élévation et son contraire s'affrontent dans la plus puissante des configurations conflictuelles. Mais le conflit des forces est déjà présent dans la bande inférieure et demeure actif, sous une forme moins intense, dans la bande supérieure, là où les élus aident d'autres élus à poursuivre leur montée.

On peut décrire la bande inférieure de la fresque selon la marque aspectuelle de l'inchoativité ; les squelettes commencent à devenir corps dans la résurrection, les damnés commencent à devenir animaux dans la damnation. L'anonymat de la plupart des personnages peints garantit et facilite la construction de ce type de processus. Sur la droite, la bande moyenne marque un progrès dans l'élévation et dans la résurrection, alors que la lutte avec les anges doit être comprise comme un moment qui précède le désir de se conformer à la justice divine. Dans la bande centrale l'aspect de l'action de résurrection approche sa détermination terminative ; les gestes d'aide, de stupeur et de joie marquent le moment même de l'acquisition du «corps ressemblant». Chez les 
anges qui portent les objets de la Passion, le devenir corps des squelettes atteint sa forme la plus parfaite dans l'assomption et le développement du mouvement du corps du Christ. Cependant, malgré la définition de ce progrès, l'action demeure dans son état imperfectif. L'ensemble des actions doit en effet se loger dans la mince épaisseur d'un temps ponctuel qui est marqué trois fois: par le geste du Christ, par le son des trompettes et par l'instant du jugement de soi ${ }^{26}$.

Le cadre formel de l'ensemble est déterminé par la coordination du temps du jugement du Christ avec le temps du jugement de soi. Le processus d'assujettissement doit correspondre au processus d'identification. Par cette articulation, le temps de l'auto/ analyse est intégré au temps du jugement final. Le temps accordé au spectateur de la fresque devient ainsi le temps d'une mémoire poussée à se ramasser en un instant par une force divine.

Le champ des forces affectives ouvert par la fresque se déploie entre les deux pôles de l'espoir et de la crainte. Chez Giotto, comme encore dans le Jugement dernier de Fra Bartolomeo, les élus sont parfaitement statiques alors que l'action de punition est en cours comme s'il y avait deux temps en un : celui des damnés ressemblant au nôtre et celui des élus éternel et immobile. Dans la fresque de Michel-Ange, la dimension processuelle des actions et des affects est partout répandue de sorte que l'opération affective se greffe sur l'aspectualité inchoative de l'ensemble. Michel-Ange fait de sa fresque une grande machine à transformer et à convertir fondée sur l'anthropologie chrétienne de la ressemblance et de la conformation et sur la participation du sujet à son salut ou à sa damnation. Cette participation se fonde sur l'auto-analyse, opération qui est pour la première fois posée au centre d'une représentation de la fin des temps. Elle est essentielle à la définition du sujet en tant que tel, aussi importante que son assujettissement par le Christ juge.

Je voudrais terminer en soulignant la place accordée aux attouchements des corps : à la lutte, comme à l'entraide et aux baisers. C'est par l'analyse de ces contacts multiples qu'on parvient à saisir la puissante dimension érotique de la fresque, au point qu'on peut être tenté de la voir comme une saisissante figuration de l'espoir d'être touché et de la crainte d'être battu. Ces affects élémentaires ont un investissement figuratif ou métaphorique qui ne parvient pas à en effacer complètement l'origine à cause de la prégnance et de la furia des postures. La chorégraphie est le schéma le plus adapté pour décrire à la fois l'organisation syntaxique des figures et l'intensité variable de leur mouvement. Le théologien Andrea Giovanni Gilio s'y réfère explicitement, bien que d'un point de vue critique et moralisateur, quand il écrit que les anges qui portent la croix et la colonne dansent une moresque ${ }^{27}$.

Un fragment d'un sonnet de Michel-Ange daté des années de la réalisation de la fresque montre que l'attente du Jugement a été conçue par lui comme l'espoir d'être transformé en quelqu'un qui plaise au Seigneur :

Signor, nell'ore streme

stendi ver me le tue pietose braccia

tormi a me stesso

e fammi un che ti piaccia

Seigneur dans l'heure extrême

tends vers moi tes bras de piété

enlève moi de moi-même

et fais de moi un qui te plaise ${ }^{28}$. 
31 Dess, comme dans la fresque, l'espoir d'être sauvé correspond à l'espoir de plaire, d'être touché et d'assumer une image christomorphe. Vision plus proche de la tradition mystique de la fulguration que de l'introspection dont, par ailleurs, d'autres sonnets de l'artiste portent le témoignage, parfois désespéré, de sorte qu'en laissant ouverte la tension entre l'analyse de soi et l'abandon à l'Autre, la fresque ouvre le champ de force dans lequel le sujet « moderne » aura à trouver sa place.

\section{NOTES}

1. Leo Steinberg, "Michelangelo Last Judgement as Merciful Heresy », Art in America novembredécembre 1975, p. 49-63, "The Line of Fate in Michelangelo's Painting ", Critical Inquiry 6 (printemps), 1980, p 411-454.

2. Sur les déterminations théoriques de l'instance du spectateur voir Meyer Shapiro, «On some Problems in the Semiotics of Visual Art : Field and Vehicle in Image-Signs », Semiotica vol. $1 \mathrm{n}^{\circ} 3$, 1969, p .223-242 ; Louis Marin, «À propos d'un carton de Le Brun: la peinture d'histoire ou la dénégation de l'énonciation ", Revue des Sciences humaines 157, 1975, p. 40-64, repris in Détruire la peinture, Paris, 1977, p. 62-69 ; id. Le portrait du roi, Paris, Minuit, 1981. J'ai proposé une analyse de la construction du spectateur dévot par trois chapelles baroques in Giovanni Careri, Envols d'Amour. Le Bernin : montage des arts et dévotion baroque, Paris, 1990. Pour le spectateur empirique et historique du Jugement dernier voir Bernardine Anne Barnes, Michelangelo's Last Judgement: The Renaissance Response, Berkeley, 1998.

3. La notion d'aspect - d'origine sémiotique et linguistique - consiste en un ensemble de catégories susceptibles de décrire la temporalité interne d'une action comme inchoative ou terminative, durative ou ponctuelle, accomplie ou inaccomplie en relation avec un observateur impliqué dans le discours. L'aspectualisation est un dispositif d'énonciation qui ne caractérise pas seulement l'expression linguistique mais aussi toute figuration du temps de l'action y compris visuelle. Voir l'entrée Aspectualisation, in A. J. Greimas, J. Courtés, Sémiotique, dictionnaire raisonné de la théorie du langage, Paris, Hachette, 1979. Voir aussi J. Fontanille (éd.), Le discours aspectualisé, Limoges, Amsterdam, Philadephia, PULIM /Benjamins, 1991 ; Francesco Marsciani, « Aspetto » in Lucia Corrain, Il lessico della semiotica (Controversie), Bologne, Esculapio, 1991, p. 135-144.

4. Pierluigi De Vecchi utilise à ce propos l'expression "sintassi dei corpi », opposant ce mode de construction de l'espace à celui de la voûte de la Sixtine sans tirer cependant de cette opposition des conclusion d'ordre sémantique et théologique comparables à celles ici proposées, cf. Pierluigi De Vecchi, «Sintassi dei corpi e modi delle attitudini dalla volta al giudizio », in Kathleen WellGarris Brandt (éd.), Michelangelo: La Cappella Sistina. Atti del Convegno Internazionale di Studi, Rome, mars, 1990, Novara, 1994, p. 201-207.

5. Giorgio Agamben, Le temps qui reste. Un commentaire de l'Epitre aux Romains (2000)traduit de l'italien par Judith Revel, Paris, Rivages, 2004. La référence aux textes de l'Apôtre en relation avec la fresque n'a pas besoin de justifications particulières s'agissant d'un élément essentiel du corpus eschatologique chrétien, voir Marcia Hall, « Michelagelo's Last Judgement : Resurrection of the Body and Predestination », Art Bulletin 58, 1976, p. 85-92.

6. « Evvi Cristo, il quale sedendo, con faccia orribile e fiera ai dannati si volge maledicendogli, non senza gran timore della Nostra Donna che, ristrettasi nel manto, ode e vede tanta rovina ». (Il y a le Christ, assis, le visage horrible et féroce adressé aux damnés, il les maudit, non sans grande crainte de 
Notre Dame qui serrée dans son manteau, entend et voit tant de ruine). Giorgio Vasari, Le Vite, éditions de 1550 et 1568.

7. "Sopra li Angioli delle trombe é il Figliol de Iddio in maiestà, col braccio e potente destra elevata, in guisa d'uomo che irato maledica i rei e li scacci dalla faccia sua al fuoco eterno, e colla sinistra distesa alla parte destra par che dolcemente raccolga $i$ buoni ", Ascanio Condivi, Vita di Michelagelo Buonarroti, (1553), Florence, 1998 p. 50. Je traduis.

8. Thessaloniciens $2,1,9$ «Ceux là subiront la peine d'une perdition éternelle, loin de la face du Seigneur et de la gloire de sa force »... Saint Paul cite Isaïe 2, 10, 19, 21.

9. Michel Foucault décrit le procès d'intériorisation comme constitutif du processus qui constitue le «sujet moderne ». Selon son analyse cependant ce processus peut être considéré pleinement opératif seulement quand l'objet de la faute (comme aussi tout mouvement fautif de l'âme aussi petit soit-il) devient objet de discours. Cette " mise en discours » de l'intériorité sera généralisée à travers la diffusion de la pratique de la confession promue par la réforme tridentine quelques années après la réalisation de la fresque. cf. Michel Foucault, Histoire de la sexualité, vol. I : La volonté de savoir, Paris, 1976. D’autres considérations importantes sur le processus de subjectivation se trouvent dans L'herméneutique du sujet : cours au Collège de France (1981-1982), éd. François Ewald et Alessandro Fontana, Paris, 2001.

10. "Sotto i piedi di Cristo i sette angeli scritti da Giovanni Evangelista, con le sette trombre che, sonando a sentenza, fanno arricciare $i$ capelli a chi li guarda per la terribilità che essi mostrano nel viso; e fra gl'altri vi son due Angeli, che ciascuno ha il libro delle vite in mano ». (Sous les pieds du Christ les sept anges décrits par Jean l'Evangéliste avec les sept trompes qui, en jouant pour annoncer la sentence, font dresser les cheveux sur la tête à celui qui les regarde pour la terribilità qu'ils montrent dans le visage, et parmi d'autres il y a deux anges, chacun le livre des vie à la main). Giorgio Vasari, Le Vite, éditions de 1550 et 1568 (je traduis).

11. «...nella parte di mezzo dell'aria, vicini alla terra sono li sette Agnoli scritti da San Giovanni nell'Apocalipse, che colle trombe a bocca chiamano i morti al giudizio dalle quattro parti del mondo, tra $i$ quali ne son due altri con un libro aperto in mano, nel quale ciascheduno leggendo, e riconoscendo la vita passata, abbia quasi da sé stesso a giudicarsi », (je traduis).

12. Origène, In epist. Romanos 9,41 Sur l'exégèse d'Origène à la Renaissance cf. Paul Oskar Kristeller, "Augustine and the Early Renaissance ", The Review of Religion VIII, 1944, p. 355-372.

13. L'absence de noms dans les pages des livres des vies est une façon d'écarter toute référence à la prédestination mais elle me semble privilégier les modèles d'intériorisation défendus à la fois par les protestants et par les catholiques réformistes par rapport à la valorisation de la médiation impliquée par la confession.

14. Agamben, op. cit, p. 128.

15. "Quaedam igitur uis est intellegenda diuina, qua fiet, ut cuique opera sua, uel bona uel mala, cuncta in memoriam reuocentur et mentis intuitu mira celeritate cernantur, ut accuset uel excuset scientia conscientiam atque ita simul et omnes et singuli iudicentur ». Augustin, De civitate Dei XX, 14.

16. "Quae nimirum vis divina libri nomen accepit. In ea quippe quodam modo legitur, quidquid ea faciente recolitur ", ibidem.

17. Cf. Brian Stock, Augustin the reader.Meditation, Self Knowledge, and the Ethics of Interpretation, Cambridge, 1996.

18. Cf. L. Steinberg, "Michelangelo's "Last Judgment" as Merciful Heresy », cité infra. note 15, p. 62 , et p. 58, qui renvoie à la doctrine d'Origène, repris par Erasme - «les tourments de l'enfer [...] ne sont rien d'autre que la perpétuelle angoisse de l'esprit liée au péché » (l'Enchiridion, 1503) et de Pomponazzi : Traité sur l'immortalité de l'âme, 1516. Dans le cercle des Spirituali (auxquels étaient liés Vittoria Colonna et Michel-Ange) animé par la pensée de Juan de Valdès, la discussion sur la nature et l'existence matérielle de l'enfer s'est développée selon la perspective d'Erasme. Une confirmation visuelle de mon interprétation est offerte par la représentation d'un damné qui cache sa figure devant le miroir que lui tend un démon dans le jugement dernier de Huys du 
musée de Beaux-Arts de Bruxelles. On peut considérer ce choix iconographique comme une explicitation de la figure michelangiolesque du désespéré.

19. Pomponazi, Trattato sull'immortalità dell'anima (1516), a cura di Vittoria Perrone Compagni, Leo. S. Olschki Editore, 1999, 59, p. 98. Je remercie Xavier Vert d'avoir attiré mon attention sur ce texte.

20. Saint Paul aux Philippiens III, 20-21, « Dominum Iesum Christum, qui transfigurabit corpus humilitatis nostrae, ut illud conforme faciat corpori gloriae suae secundum operationem, qua possit etiam subicere sibi omnia», je souligne.

21. Inferno, III, 121/126, trad. J. Risset.

22. Ascanio Condivi, Vita di Michelangelo Buonarroti, (1553), Florence, 1998 p. 50, je traduis. « Sotto ai quali reprobi si vede Caronte colla sua navicella, tal quale lo descrive Dante nel suo Inferno, nella palude dell'Acheronte, il qual alza il remo per battere qualunque anima lenta si dimostrasse. E giunta la barca alla ripa, si veggion tutte quell'anime dalla barca a gara gittarsi fora, spronate dalla giustizia divina, si che la tema, come dice il poeta, si volge in desio... ».

23. Pierluigi De Vecchi, «Sintassi dei corpi.. », art. cit.

24. Alexander Nagel, Michelangelo and the reformation of the art, New York, 2000.

25. Giovan Paolo Lomazzo, Trattato dell'arte della pittura, scoltura et architettura, Milan, 1584 : «Dicesi adunque che Michelangelo diede una volta questo avvertimento a Marco da Siena, pittore suo discepolo, che dovesse sempre fare la figura piramidale, serpentinata e moltiplicata per uno, doi e tre. Et in questo precetto parmi che consista tutto il secreto de la pittura. Imperoché la maggior grazia e leggiadria che possa avere una figura é che mostri di moversi, il che chiamano i pittori furia della figura. E per rappresentare questo moto non vi é forma più accomodata, che quella della fiamma del foco, [...] ha il pittore d'accompagnare questa forma piramidale con la forma serpentinata, che rappresenta la tortuosità di una serpe viva quando cammina, che é la propria forma de la fiamma del foco che ondeggia».

26. Inchoatif, terminatif, imperfectif, ponctuel, sont autant d'aspects de l'action selon la terminologie de la linguistique et de la sémiotique.

27. Giovanni Andrea Gilio, "Degli errori e degli abusi dei pittori circa l'istorie ", in Paola Barocchi (éd.), Scritti d'arte del cinquecento (1564), vol. 4, Turin, 1978, p. 851.

28. Carl Frey, Die Dichtungen des Michelagniolo Buonarroti, Berlin, 1897, $\mathrm{n}^{\circ}$ LXXXVII.

\section{INDEX}

Mots-clés : Michel-Ange, Chapelle Sixtine, transformation, spatialité

\section{AUTEUR \\ GIOVANNI CARERI}

Giovanni Careri est directeur d'études à l'EHESS où il dirige le Centre d'Histoire et Théorie des Arts (CEHTA). Parmi ses publications, Envols d'amour. Le Bernin montage des arts et dévotion baroque, Usher, Paris, 1990, La Jérusalem délivrée du Tasse. Poésie, peinture, musique, ballet, Klincksieck, Paris, Musée du Louvre, 1999 (édition des actes du colloque international organisé à l'Institut Culturel Italien et au Louvre en 1993), Baroques, Paris, Citadelles \& Mazenod, 2002 (Traduction anglaise : Baroques, Princeton University Press, 2003. Traduction italienne : Il Barocco nel mondo, Florence, Le 
Lettere, 2003). Gestes d'amour et de guerre. La Jérusalem délivrée, images et affects (XVI ${ }^{e} \mathrm{XVIII}^{e}$ ), Paris, Editions de l'EHESS, 2005 (à paraître en traduction italienne chez Il Saggiatore, Milan).

Giovanni.Careri@ehess.fr 